\title{
Proteomic analysis highlights the role of detoxification pathways in increased tolerance to Huanglongbing disease
}

\author{
Federico Martinelli ${ }^{1}$, Russell L. Reagan², David Dolan², Veronica Fileccia ${ }^{1}$ and Abhaya M. Dandekar²*
}

\begin{abstract}
Background: Huanglongbing (HLB) disease is still the greatest threat to citriculture worldwide. Although there is not any resistance source in the Citrus germplasm, a certain level of moderated tolerance is present. A large-scale analysis of proteomic responses of Citrus may help: 1) clarifying physiological and molecular effects of disease progression, 2) validating previous data at transcriptomic level, and 3) identifying biomarkers for development of early diagnostics, short-term therapeutics and long-term genetic resistance.

Results: In this work we have conducted a proteomic analysis of mature leaves of two Citrus genotypes with well-known differing tolerances to HLB: Navel orange (highly susceptible) and Volkameriana (moderately tolerant). Pathway enrichment analysis showed that amino acid degradation processes occurred to a larger degree in the Navel orange. No clear differences between the two genotypes were observed for primary metabolic pathways. The most important finding was that four glutathione-S-transferases were upregulated in Volkameriana and not in Navel orange. These proteins are involved in radical ion detoxification.
\end{abstract}

Conclusions: Upregulation of proteins involved in radical ion detoxification should be considered as an important mechanism of increased tolerance to HLB.

Keywords: Citrus, Huanglongbing, Candidatus liberibacter, iTRAQ, Proteome, Proteomic

\section{Background}

Huanglongbing disease currently threatens areas where Citrus cultivation is important in the agricultural economy such as East Asia, the Middle East, and the Americas. Huanglongbing disease is caused by three species of Candidatus liberibacter asiaticus (CaLas), americanus and africanus [1]. The pathogen is transmitted by two species of psyllids: Diaphorina citri and Trioza erytreae. Recently, Trioza erytreae was found for the first time in Europe (Galicia, Spain). Typical symptoms of Huanglongbing disease in leaves include shoot yellowing and blotchy, mottled leaves. Although most of the fruits are still of commercial quality, fruits from severely affected branches are unmarketable: small, lopsided, green, and acidic, with many aborted seeds. Leaves accumulate starch, phloem is damaged and cell wall lamellae swell during CaLas

\footnotetext{
* Correspondence: amdandekar@ucdavis.edu

${ }^{2}$ Plant Sciences Department, University of California, One Shields Avenue, 95616 Davis, CA, USA

Full list of author information is available at the end of the article
}

infection [1,2]. Candidatus liberibacter spp. belong to the alpha subdivision of the proteobacteria based on ribosomal region sequence data [3]. The bacterium has not yet been definitively cultured despite attempts to do so [4]. Koch's postulates have not been fulfilled for this disease, so possible interactions with other microrganisms cannot be ruled out. The pathogen lives in the insect and in the phloem of Citrus trees. Once acquired, it typically persists for the rest of the life of the host. Insecticides can decrease psyllid populations, but since the pathogen remains in the vector, disease spread can occur with the presence of just a few infected psyllids in the orchard. All genotypes within the genus Citrus are susceptible to HLB to varying degrees although species of other close-related genera showed some sort of resistance [5]. There is variability in disease severity and symptoms among Citrus genotypes [6]. Murraya paniculata (orange jasmine), an ornamental Citrus closely-related plant, showed fewer symptoms of the disease [7]. A study examining the responses of 30 genotypes to HLB disease grouped them based on phenotypic 
analysis of induced symptoms [5]. Another recent study has evaluated 65 Citrus accessions and 33 accessions belonging to other closely related genera. Resistance was reported in accessions not belonging to Citrus genera [8]. Another work have screened Citrus germplasm susceptibility to HLB analyzing sixteen Citrus genotypes [9]. Results showed that Citrus macrophylla and C. medica were the most susceptible while complex genetic hybrids 'US 1-4-59' and 'Fallglo' were the least susceptible. A metabolomic investigation was conducted comparing five different tolerant hybrids and a highly susceptible cultivar to identify potential metabolites linked with diverse response [10]. The causes of the disease have been studied using different "omic" approaches to identify which genes, proteins and metabolites may be targeted by innovative diagnostic and therapeutic methods. The genome of the pathogen was sequenced using a metagenomic approach, both from infected plants [11] and the insect vector [12]. No toxins or other secreted proteins have been linked to the disease and the mechanisms of its pathology are still unclear. Large scale microarray analysis revealed significant modulation of genes involved in transport, cell defense and carbohydrate metabolism [13, 14]. Photosynthesis is diminished in both young and mature leaves, but it is upregulated in infected fruits [14]. Starch accumulation was linked to the upregulation of genes involved in glucose import into the chloroplast and starch biosynthesis $[15,16]$. A modulated Jasmonic (JA)-Salicilic acid (SA) crosstalk of innate responses may lead to a misdirected defence response. An integrated approach of 2-DE and mass spectrometry showed that changes in levels of several proteins involved in photosynthesis and protein synthesis were linked to reduced concentrations of $\mathrm{Ca}$, $\mathrm{Mg}, \mathrm{Fe}, \mathrm{Zn}, \mathrm{Mn}$ and $\mathrm{Cu}$ in infected grapefruit leaves [17]. Proteins upregulated in infected samples were involved in redox stage and cell defense such as $\mathrm{Cu} / \mathrm{Zn}$ superoxide dismutase, peroxidases, chitinases and lectin-related proteins [18]. 'Madam Vinous' sweet orange plants infected by CaLas showed increased miraculin-like proteins, chitinase, $\mathrm{Cu} / \mathrm{Zn}$ superoxide dismutase and lipoxygenase. Some key metabolites modulated by HLB include proline, $\beta$-elemene, (-)-trans-caryophyllene, and $\alpha$-humulene [19]. Increased accumulation of some amino acids (L-proline, L-serine, and L-aspartic acid) and organic acids was linked to greater susceptibility of 'Madam Vinous' sweet orange compared to Carrizo citrange [20]. However, it is worthy to notice that these trees were highly infected with many secondary effects so it will be necessary to confirm these results with newly infected trees. An increased amount of most amino acids, involved in plant defense to pathogens was observed in tolerant varieties in such as phenylalanine, tyrosine, tryptophan, lysine, and asparagine [21].

This study examines proteomic changes in fully photosynthesizing leaves to determine how disease mechanisms and susceptibility vary between two Citrus genotypes, using an integrated approach of principal component analysis (PCA), gene set and pathway enrichment analysis. The purpose was to characterize key proteins and posttranscriptionally modulated pathways in different Citrus genotypes at a late symptomatic stage of HLB.

\section{Results and discussion}

Navel orange (Citrus sinensis (L.)) is an HLB-sensitive cultivar while Volkameriana is moderately tolerant [5]. Different techniques (2-DE, mass spectrometry and ICP mass spectroscopy) have been used to identify key proteins differentially regulated by HLB in Citrus leaves $[19,20]$. Over 4000 proteins were analyzed using isobaric tags for relative and absolute quantitation (iTRAQ) for both genotypes (4557 in Volkameriana and 4521 in Navel orange). In Navel orange, 599 proteins were differentially regulated between infected and healthy tissue (P-value $<0.05$ and $\log _{2} \mathrm{FD}>0.5$ and $<-0.5$ ) (Additional file 1: Table S1). In Volkameriana, 411 differentially regulated proteins were found between infected and healthy tissue (Additional file 2: Table S2).

\section{PCA analysis}

PCA was used to visualize differences between the four analyzed genotypes $\mathrm{x}$ disease status, subdividing the entire proteomic profile into three important subcategories: biotic stress responses, overall cell metabolism and transcriptional regulation pathways (Fig. 1). All identified proteins belonging to these important functional categories were used for the PCA plots. The four categories of samples (healthy $\mathrm{V}$, healthy $\mathrm{N}$, infected $\mathrm{V}$ and infected $\mathrm{N})$ were clearly separated in all three PCA plots, implying significant protein changes in all three gene categories related to species and health status. For biotic stressrelated proteins, PC1 and PC2 accounted for 40 and $24 \%$ of the data variability, respectively. Important proteins associated with each functional category that contributed to separation between sample types (indicated by directions of vectors) are listed in Fig. 1. Some key proteins involved in redox state significantly contributed in the separation of Infected V from the rest of the other categories. In the general metabolism PCA plot, the PC1 and PC2 accounted for 49 and $24 \%$ of the data variability, respectively. Key proteins involved in primary metabolism associated with the separation of Healthy $\mathrm{V}$ from the Infected $\mathrm{V}$ include malate dehydrogenase and pyruvate dehydrogenase (TCA cycle-glycolysis), sucrose synthase and AGPase (sucrose and starch metabolism). The third PCA plot was generated based on the expression of proteins involved in transcriptional regulation, signaling, hormone and redox state. PC1 and PC2 accounted for 37 and $27 \%$ of data variability, 


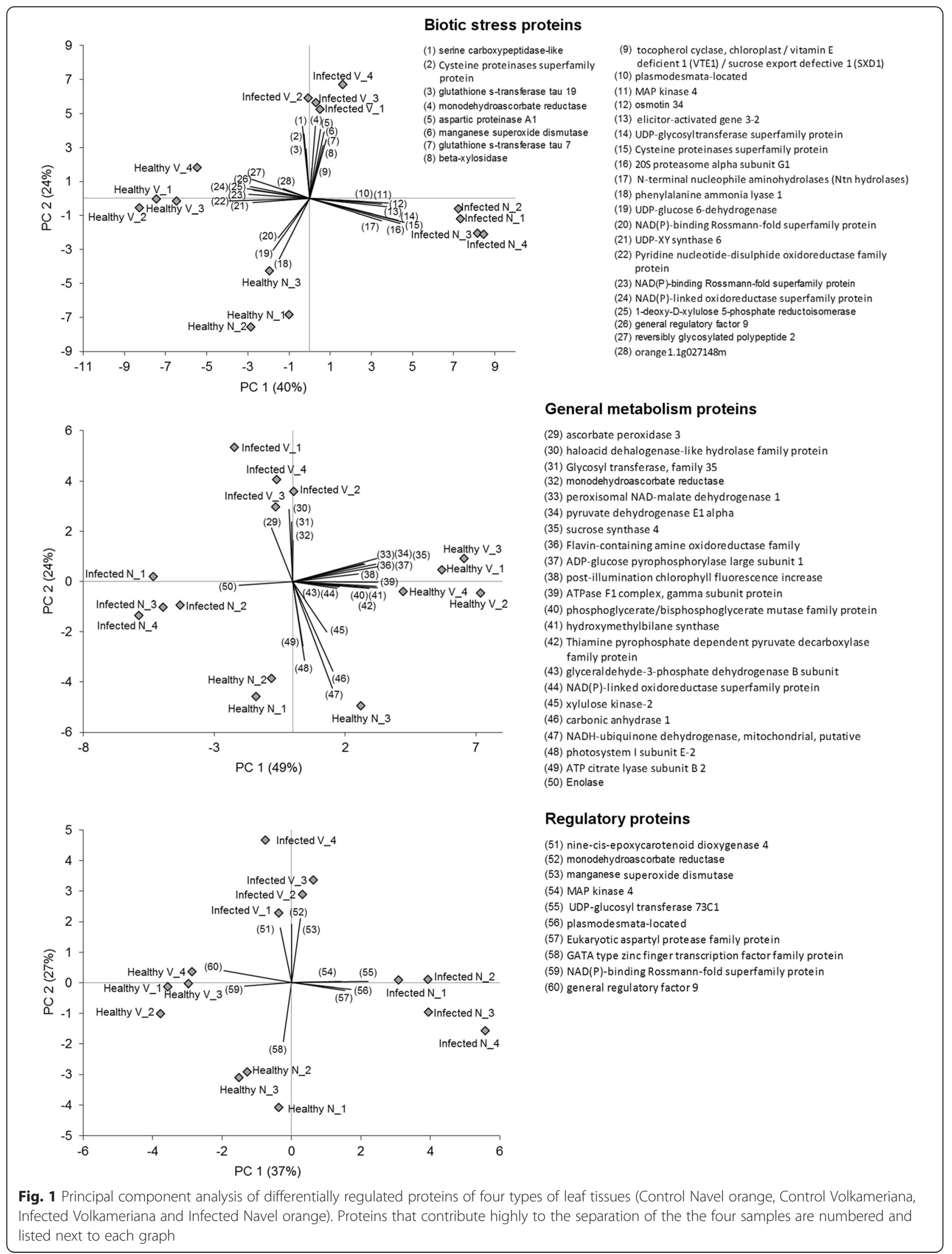


respectively. Interestingly, the regulation of few important proteins seems to specifically characterize the infected vs. healthy state of Navel orange. These include MAP kinase 4, UDP-glucosyl transferase, and aspartyl protease. No changes were observed for MAPK6. Proteins that appear in the three PCA plots were highly regulated in the comparison between infected and control in both Citrus genotypes. Indeed, they may be considered as putative candidate biomarkers of a clear symptomatic status in Citrus at proteomic level. Their HLB-regulated pattern of expression greatly contributed in distinguishing the four different leaf sample types. Further analysis will be needed to validate these data and confirm the role in the pathogenesis of HLB disease.
Gene set and pathway enrichment analysis

The Pageman web tool highlighted which gene categories were up- or down-regulated in each pairwise comparison (Fig. 2). Both infected genotypes exhibited repressed amino acid biosynthesis and protein synthesis. In Navel orange, photosynthesis, isoflavone pathways, tetrapyrrole synthesis, and galactose metabolism were significantly inhibited by HLB. In Volkameriana, $\mathrm{S}$-assimilation, isoprenoids, RNA binding and amino acid activation were specifically diminished. In both genotypes, HLB enhanced starch-related pathways, biotic stressrelated proteins, beta 1,3 glucan hydrolases, and protein degradation pathways. Some distinct differences were observed between genotypes in HLB response. In Volkameriana, cell wall modifications, galactose metabolism,

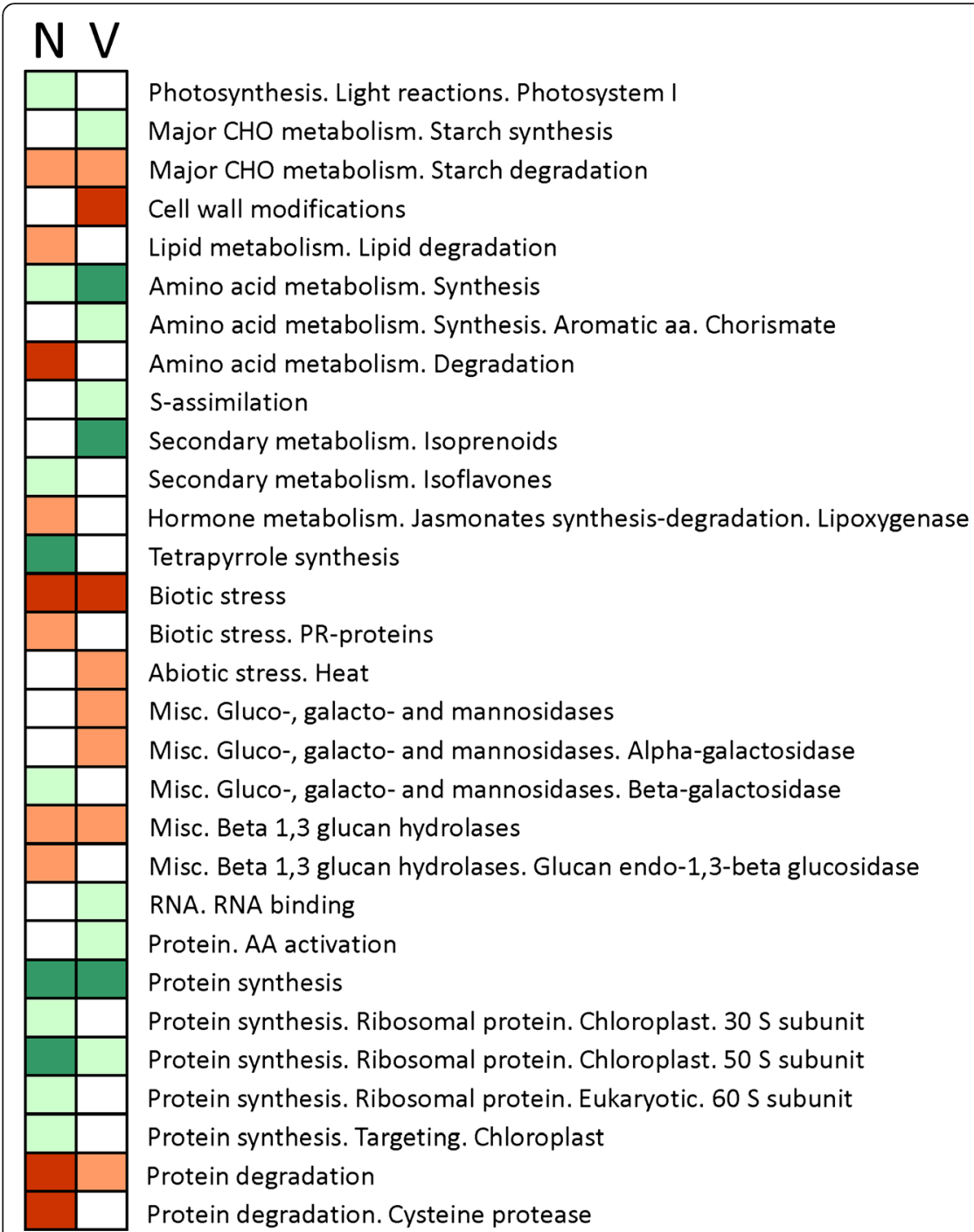

Upregulated in infected samples

$\square$ Log fold ratio $\geq 3$

Log fold ratio $>0.5$ and $<3$

Downregulated in infected samples

Log fold ratio $\leq-3$

Log fold ratio $<-0.5$ and $>-3$

Not significantly regulated in infected samples

Fig. 2 Gene set enrichment analysis using Pageman web-tool. Upregulated and downregulated pathways at proteomic level in infected Volkameriana $(\mathrm{V})$ and infected Navel orange $(\mathrm{N})$ in comparison to respective healthy controls 
and heat shock proteins were upregulated. In Navel orange, amino acid degradation, lipid metabolism, jasmonates, and PR-proteins were upregulated.

A pathway enrichment analysis was performed using the DAVID bioinformatic resource to determine which metabolic pathways were commonly or specifically affected by HLB disease in both genotypes (Table 1). Some metabolic pathways were altered by HLB in both species. Amino acid metabolism (glycine, serine, threonine, phenylalanine and tryptophan) was significantly downregulated. Other key inhibited pathways include biosynthesis of plant hormones, terpenoids, and phenylpropanoids. On the other hand, tyrosine metabolism was upregulated in both genotypes. In Navel orange, fatty acid biosynthesis and nitrogen metabolism were diminished while alphalinolenic acid metabolism was enhanced. In Volkameriana, alkaloids and pyruvate metabolism-related proteins were repressed while galactose metabolism and fatty acid metabolism were upregulated.

\section{Primary metabolism}

The integration of the two pairwise comparisons into a unified Mapman visualization allowed us to identify proteins that were commonly or specifically regulated in response to HLB in the two species. Some proteins involved in cell wall modifications were upregulated by HLB only in Volkameriana: expansin A1, expansin A8, expansin-like B1, xyloglucan endotransglycosylase 6 , and xyloglucanxyloglucosyltransferase (TCH4) (Additional file 3: Figure S1). In Navel orange, proteins involved in fatty acid biosynthesis and elongation were generally repressed while several key proteins involved in amino acid degradation were upregulated: arginase, pyrroline-5-carboxylase reductase, lactoylglutathione lyase, and 3-hydroxylmethylglutaryl-CoA lyase. The increase of protein degradation indicates that senescence processes may be more highly activated in HLB-diseased Navel orange than in Volkameriana.

Sucrose metabolism was only slightly affected by HLB at the protein level; only sucrose synthase was repressed. Starch metabolism was more altered (Fig. 3a). The first enzyme of starch biosynthesis, ADP-glucose pyrophosphorylase, was inhibited by HLB in both genotypes. In Volkameriana, starch synthase was upregulated while 1,4-alpha-glucan starch branching enzyme was slightly downregulated. Among starch degradation enzymes, glucan phosphorylase and heterogycan glucosidase 1 were upregulated in both genotypes. Alpha-amylase was upregulated in Volkameriana while beta-amylase 6 was enhanced in Navel orange. Taken together these findings showed that starch metabolism was highly affected in both genotypes at the protein level. No clear association between differing susceptibility to HLB is evident from starch pathway regulation alone.

A significant downregulation in HLB-infected samples was observed for proteins involved in photosynthetic reactions [17]. The altered transcription of sugar and starch metabolism genes caused by HLB [16] mostly agreed with the corresponding protein changes presented in the present

Table 1 Pathway enrichment analysis using DAVID Bioinformatics Resources 6.7. Pathways that were upregulated and downregulated for the healthy/infected comparison for each genotype are indicated with the corresponding $p$-value

\begin{tabular}{|c|c|c|c|c|}
\hline \multirow[t]{2}{*}{ Pathway } & \multicolumn{2}{|c|}{ Navel orange } & \multicolumn{2}{|c|}{ Volkameriana } \\
\hline & Up & Down & Up & Down \\
\hline Fatty acid biosynthesis & & $9.2^{*} 10^{-3}$ & & \\
\hline Glycine, serine and threonine metabolism & & $1.3^{*} 10^{-2}$ & & $2.6^{*} 10^{-2}$ \\
\hline Biosynthesis of plant hormones & & $1.5^{*} 10^{-2}$ & & $1.4^{*} 10^{-3}$ \\
\hline Porphyrin and chlorophyll metabolism & & $1.6^{*} 10^{-2}$ & & $2.9^{*} 10^{-2}$ \\
\hline Phenyalanine, tyrosine and tryptophan biosynthesis & & $2.7^{*} 10^{-2}$ & & $3.3 * 10^{-3}$ \\
\hline Carbon fixation in photosynthetic organisms & & $2.9^{*} 10^{-2}$ & & \\
\hline Terpenoid backbone biosynthesis & & $3.0^{*} 10^{-2}$ & & $2.1^{*} 10^{-2}$ \\
\hline Nitrogen metabolism & & $3.3^{*} 10^{-2}$ & & \\
\hline Biosynthesis of phenylpropanoids & & $4.8^{*} 10^{-2}$ & & $2.3^{*} 10^{-2}$ \\
\hline alpha-linolenic acid metabolism & $1.6^{*} 10^{-3}$ & & & \\
\hline Tyrosine metabolism & $8.3^{*} 10^{-3}$ & & $1.8^{*} 10^{-2}$ & \\
\hline Biosynthesis of alkaloids from shikimate pathway & & & & $4.4^{*} 10^{-3}$ \\
\hline Pyruvate metabolism & & & & $2.5^{*} 10^{-2}$ \\
\hline Biosynthesis of alkaloids from terpenoid and polyketide & & & & $4.5^{*} 10^{-2}$ \\
\hline Proteasome & & & $2.7^{*} 10^{-3}$ & \\
\hline Galactose metabolism & & & $8.2^{*} 10^{-3}$ & \\
\hline Fatty acid metabolism & & & $4.3^{*} 10^{-2}$ & \\
\hline
\end{tabular}




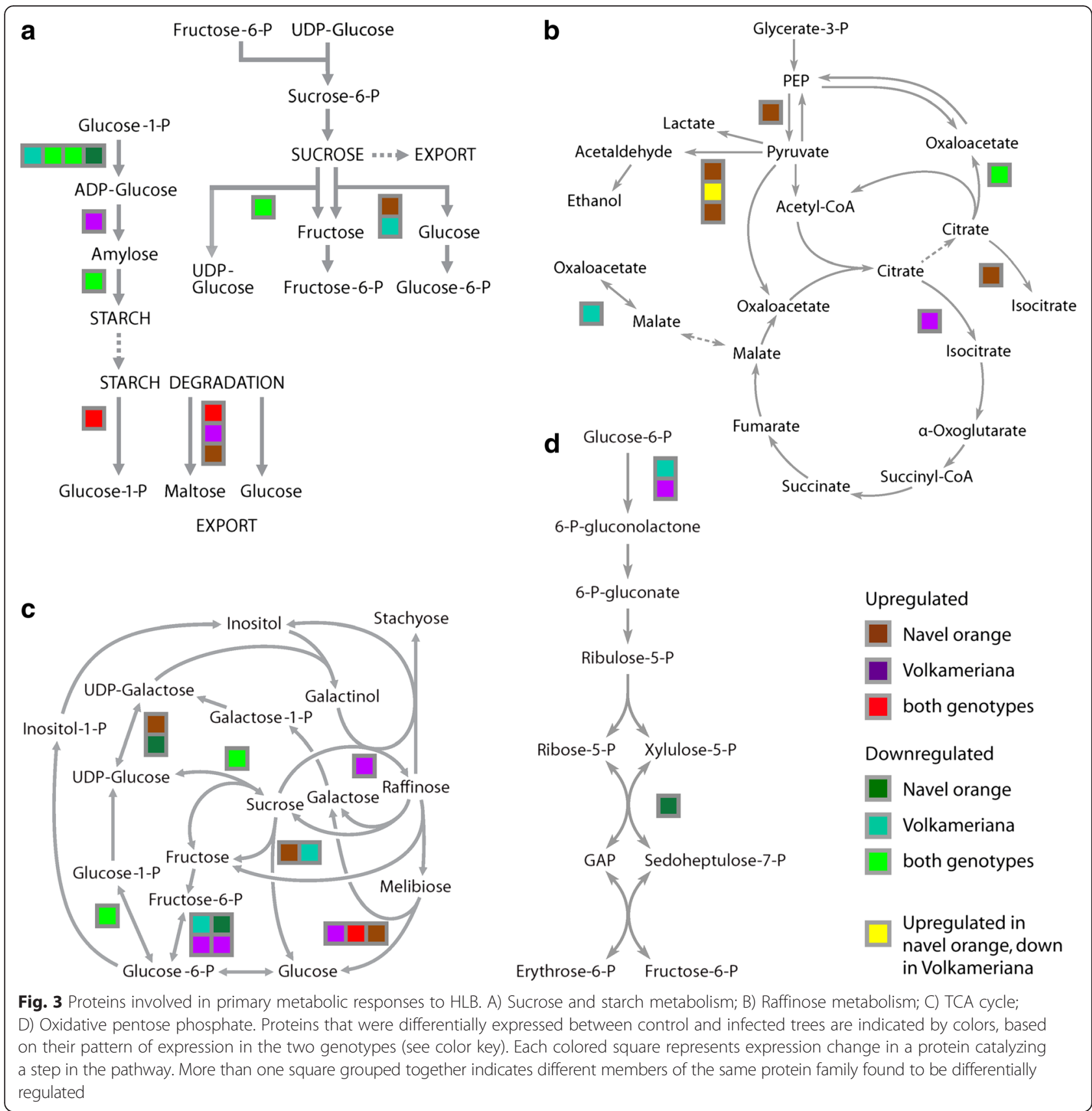

work. The observed changes in starch-related pathways were consistent with the transcriptomic analysis [16]. Increased starch degradation was observed probably due to the increased starch concentrations in infected leaf tissues. ADP glucose-pyrophospholyase (ADPase) was repressed in both infected species. This enzyme is rate-limiting for starch biosynthesis, catalyzing the conversion of glucose-1phosphate to ADP-glucose that is polymerized into amylopectin or amylose [22]. Starch accumulation in infected leaves is a typical symptom of HLB [1]. However, the greatest occurrence of this process may occur at an early, asymptomatic stage. Indeed we may speculate that the accumulation of starch may be a secondary effect of the disease instead of being the cause of symptoms. When symptoms are already evident and yellowing is present, starch biosynthesis may slow down and starch degradation is expected to be activated as a response of the plant to limit damage to cell structures. However, some differences in primary metabolism were observed between transcriptomic and proteomic approaches. Changes in invertase gene expression were observed [16], but not its protein levels. Sucrose, the substrate of invertase, is produced by photosynthesis in leaves and then transported to sink tissues (immature fruits and young leaves) through the 
phloem. Involvement of sucrose in signaling of innate responses has been described recently [23]. Invertase plays an important role in plant stress responses, possibly serving as an extracellular signal of pathogen attacks [24]. It is possible that expression of this gene in response to HLB depends on genotype, plant physiological conditions and age, tissue developmental conditions, type of infection (psyllid inoculation or graft-mediated) and/or environmental conditions (field or controlled environment). Taken together all these primary metabolism results concur with previous findings showing significant modification of transcript abundance in minor carbohydrate metabolism [16] but do not suggest a clear link to the well-known difference in tolerance between the two genotypes.

GPT2 has been linked with HLB disease: this gene is responsible for glucose import into the chloroplast and consequently for starch accumulation $[13,16]$. The protein was not found among those extracted and characterized by iTRAQ, therefore no conclusions can be made about changes in protein levels due to HLB. However, the protocol used to analyze the Citrus proteome favored detection of soluble cell proteins over membrane proteins such as GPT2.

Expression of key proteins involved in the TCA cycle and PEP metabolism were affected by HLB. ATP-citrate lyase subunit B2 was repressed in both species (Fig. 3b). Alcohol dehydrogenase 1 which converts aceltaldehyde to ethanol, was upregulated in both genotypes. In infected Navel orange, isopropyl malate isomerase, which converts citrate to isocitrate and pyruvate decarboxylase involved in fermentation, was more abundant than in healthy tissue. In Volkameriana, pyruvate decarboxylase was repressed while aconitate hydratase involved in TCA cycle was upregulated.

Raffinose metabolism was drastically altered by the disease (Fig. 3c). In Navel orange, expression of alphagalactosidase 1, UDP-glucose-4-epimerase, glucose-6phosphate isomerase, and alpha-galctosidase 1 were enhanced by HLB. In Volkameriana, raffinose synthase, phosphofructokinase 3 and phosphoglucomutase were upregulated. Sucrose synthase 4 and phosphoglycerate mutase were donwregulated in both genotypes. A transketolase that converts the xylulose-5-P in sedoheptulose7-P was repressed in infected Navel orange (Fig. 3d).

Significant repression of aspartate biosynthesis and serine metabolism was observed in infected Navel orange leaves. This agrees with a previously described downregulation of serine-type peptidases at both asymptomatic and symptomatic stages [18]. In infected Navel orange, seventeen proteins involved in amino acid biosynthesis were downregulated. Fewer proteins were downregulated in infected Volkameriana. On the other hand, some upregulated proteins involved in amino acid degradation were identified only in infected Navel orange. Taken together, these findings suggest that amino acid metabolism in Navel orange is more sensitive to degradation during HLB infection than in Volkameriana.

\section{Secondary metabolism}

A general repression of key proteins involved in biosynthesis of secondary metabolites was observed in both cultivars in response to CaLas infection (Additional file 4: Figure S2). Geranylgeranyl reductase and 1-deoxyD-xylulose 5-phosphate reductoisomerase, involved in the non-MVA pathway, were repressed. Other commonly HLB-downregulated proteins involved in the shikimate pathway included 2-dehydro-3-deoxyphosphoheptonate aldolase, 3-dehydroquinate synthase, 3-phosphoshikimate1-carboxylvinyltransferase and mevalonate diphosphate decarboxylase. Two key proteins involved in phenylpropanoids, phenylalanine ammonia lyase and aryl-alcohol dehydrogenase, were more abundant in both species in response to HLB, while cinnamyl alcohol dehydrogenase 9 was repressed. Two proteins involved in alkaloid biosynthesis, tropinone reductase and strictosidine synthase-like 4, were upregulated in infected Volkameriana.

Findings related to Citrus activated defense responses against CaLas infection are shown in Fig. 4). Three proteins involved in auxin signal transduction were activated in infected Volkameriana: auxin resistant 1 and two aldo/keto reductases. One aldo/keto reductase was HLB-regulated in both genotypes. Three proteins involved in jasmonic and salicylic acid responses were induced in Navel orange but not in Volkameriana. Lipoxygenase 2 was upregulated in both genotypes.. Some key proteins involved in cell wall modifications were commonly regulated by both genotypes: UDP-glucose-6-dehydrogenase, UXS6, UXS2, and RHM1. Proteolytic-related proteins were altered in both genotypes. Taken together these findings do not suggest any clear association between the two genotypes and proteomic changes in hormonal crosstalk, cell wall and proteolytic pathways.

Some proteins synthesizing volatiles via the phenylpropanoid and carotenoid pathways were affected in the present study (Fig. 5). The marked differences between the two species suggests that to be effective, any innovative HLB-detection system based on induced volatiles must be cultivar-specific.

Plant phenols not only counteract reactive oxygen species and pathogen-secreted toxins, but also play roles in transport and signal transduction pathways. Polyphenol chemistry is critical to adapting plants to environmental stresses, including pathogens [25]. Phenylalanine ammonialyase (PAL) was downregulated by HLB infection in both species (Additional file 4: Figure S2). This enzyme is a key regulatory point for the entire phenylpropanoid pathway. Enhancement of its transcript abundance is linked to phytopathogen attacks [26]. Another important 


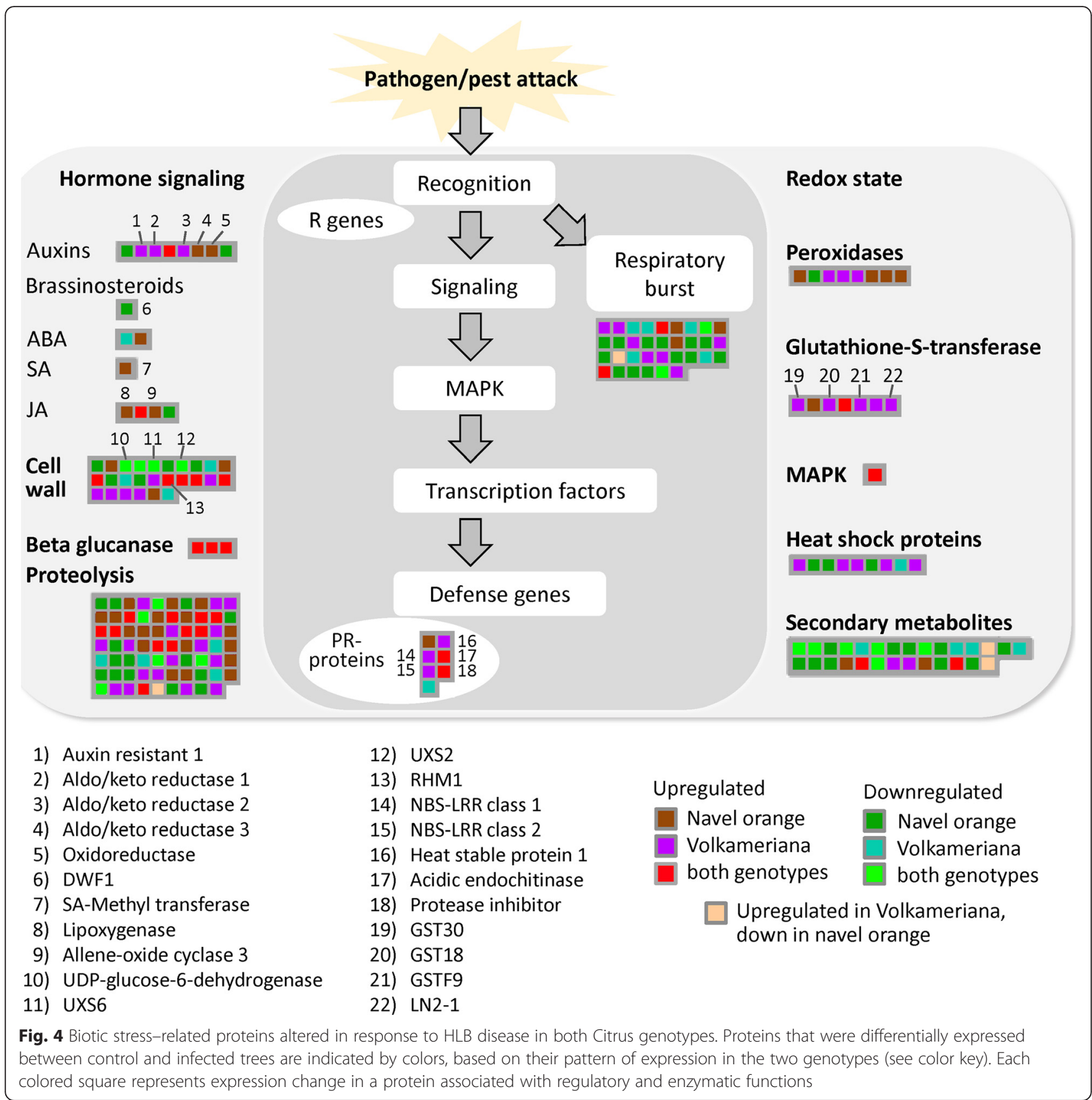

protein encoding isoflavone reductase and involved in antioxidant reactions was repressed in both the infected species, consistent with previous proteomic analysis [18]. In general, RNA-seq analysis showed that phenylpropanoid pathways were transcriptionally affected by HLB in both young and mature infected leaves in field grown mature trees [16]. These previous findings are not completely consistent with the present proteomic data. Gene set enrichment analysis showed a general downregulation of secondary metabolism in both genotypes, although some key proteins were upregulated in response to the disease. These contrasting findings may reflect differences in developmental and physiological stages of the plants analyzed in the two studies or differences in environmental and agronomic conditions. Although phenylpropanoids may be activated at early stages of infection, their repression when symptoms are severe is expected. The large number and complexity of metabolites belonging to phenylpropanoid pathways makes the clarification of their many roles in the host-pathogen battle difficult.

\section{Hormonal crosstalk}

Plant innate responses are finely controlled by hormonal crosstalk, particularly between JA and SA signaling. The 


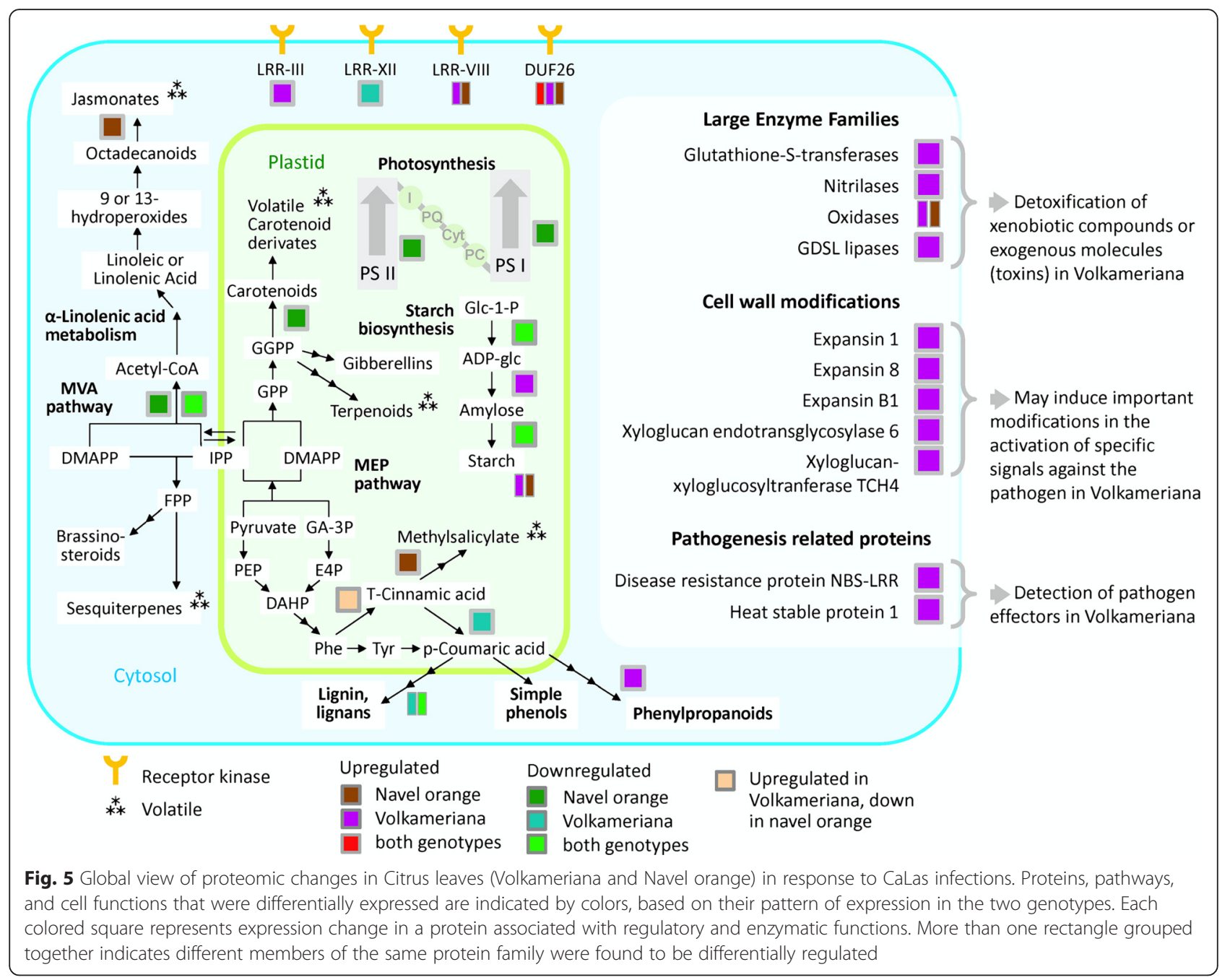

induction of allene oxide cyclase in infected Navel orange (Fig. 4) is intriguing because jasmonic acid signal transduction is a key pathway activated in response to necrotroph and herbivore attacks. JA-responsive proteins, gibberellin signaling (GASA1 and gibberellin-2-oxydase), and auxin signaling (CYP711A1 and SAUR-like proteins) were upregulated in mature infected leaves of Valencia orange [16]. Because CaLas is a biotroph, the activation of JA may be deleterious for the host as previously suggested [16]. Brassinosteroids affect disease resistance in plants [27] probably due to the induction of BAK1, which interacts with PAMP receptors such as bacterial flagellin to activate immune responses [28]. The ST1 gene was repressed in HLB-infected leaves [16]. However, the connections of brassinosteroids with the SA-ET-JA crosstalk and plant immunity remain elusive. On the other hand, auxinrelated proteins were upregulated by HLB in both Citrus genotypes. Because of the antagonist role of auxins toward SA response [29], we may speculate that these effects are deleterious to the infected host.
Proteomic studies have revealed post-transcriptional regulation of genes involved in key pathways which may be responsible for variations in phenotypic responses to HLB. The regulation of carbohydrate metabolism (sucrose, starch, and raffinose metabolism) is clearly altered at symptomatic stage at both transcript and protein levels. Differences in signaling mechanisms and hormone-mediated defense responses may also contribute to the range of tolerance to HLB within the Citrus germplasm. The most compelling finding was that proteins involved in redox pathways and defense against xenobiotics (especially GSTS) were more abundant in Volkameriana than in Navel orange, and this may be linked with the former's greater tolerance. Four GSTs were significantly upregulated in infected Volkameriana and not in infected Navel orange: GST18, GST19, GHST30, LN2-1. These effects on the proteome may explain the greater susceptibility of Navel orange compared to Volkameriana. While these findings regarding Citrus responses are valuable in the ongoing efforts to combat this deadly disease, further studies are still 
needed to validate these findings and deliver effective targets to develop new therapeutic strategies.

\section{Signaling and defense response pathways}

Some proteins encoding receptor kinases of the LRR type were HLB-regulated: one was upregulated in Volkameriana, one was repressed in Navel orange and two different proteins of LRR-VIII were upregulated (one in Volkameriana and one in Navel orange). Leucine-rich repeat receptor kinases are the largest category of receptor kinases and mediate signaling of defense responses in plants. Other receptor kinases (VIII and DUF26) were upregulated in both Citrus genotypes. The proteins belonging to Domain of Unknown Function 26 were upregulated in response to HLB: one in Volkameriana, one in Navel orange and one commonly regulated. DUF26 is one of the largest classes of receptor-like kinases (RLKs). These proteins play important roles in regulating pathogen defense and programmed cell death [30]. Based on the proteomic results in this study, we speculate that diverse signaling mechanisms occur depending on the Citrus genotype. It is possible that variability in susceptibility of Citrus may result from pathogen perception due to activation of different receptors which in turn activate defense responses which vary in speed and intensity. Much remains to be learned regarding which receptor family is involved in susceptible or resistant responses to HLB.

Several proteins involved in calcium regulation were less abundant in infected leaves of Navel orange: calmodulin-binding and calcium-transporting ATPase. This is consistent with the significant drop in calcium concentration observed in symptomatic leaves [17]. Three 14-3-3 proteins were also HLB-downregulated in Navel orange and not in Volkameriana. These are a large family of proteins present in all eukaryotic organisms that aid signaling by binding other proteins such as kinases, phosphatases, and receptors (i.e. the P-type H+ ATPases) [31].

Volkameriana showed significant stimulation of respiratory burst and consequent redox state, a prerequisite of the upregulation of pathogenesis-related proteins. Enzymes involved in the control of reactive oxygen species were generally enhanced in response to CaLas infection in both genotypes although Volkameriana showed a higher activation of glutathione-S-transferases (GST30, GST18, GSTF9, LN2-1). The upregulation of these important detoxification proteins may be linked with the increased tolerance of Volkameriana in comparison to Navel orange. An upregulation of enzymes involved in the biosynthesis of peroxiredoxins, $\mathrm{Cu} / \mathrm{Zn}$ superoxide dismutase and $2 \mathrm{Fe}-2 \mathrm{~S}$ ferredoxin-like protein, occurred at both asymptomatic and symptomatic stages [18]. Glutathione S-transferase family proteins include several isozymes that help detoxify xenobiotic compounds [32]. Plant GSTs add glutathione to electrophilic xenobiotic molecules pushing them into the cell vacuole. Regulation of these proteins by environmental stress stimuli suggests a role in protection against any harmful event [33]. GSTs have been linked with hormone homeostasis and their high affinity for auxins suggests their upregulation is a general signal of responses to stress [34]. Stress-inducible GSTs conjugate deleterious metabolites caused by oxidative damage. Inducible GSTs may play the important role of detoxifying exogenous molecules such as phytotoxins produced by pathogen attacks. Higher levels of GSTs in Volkameriana strengthens the hypothesis that they protect against dangerous molecules generated by CaLas attack. Indeed, differential activation of GSTs may explain some of the variability of Citrus responses to HLB. Some peroxidases were also upregulated in both genotypes. These enzymes detoxify excess $\mathrm{H}_{2} \mathrm{O}_{2}$ [35] and they are grouped based on their subcellular localization [36]. As previously suggested [37, 174], greater Citrus susceptibility to HLB may be linked to a failure to rapidly induce antioxidant components to alleviate the devastating effects of ROS produced by CaLas. It has been suggested [38] that this category of proteins may be considered candidate markers in fieldgrown for the detection of the devastating disease "Esca" in grapevine. In the same way high expression of GSTs are potential candidate markers for genotypes with useful tolerance to HLB. Further investigations will need to be conducted for a large number of genotypes.

Pathogenesis-related (PR) proteins are plant defensive proteins against biotic attacks [39]. More PR-proteins were induced in Volkameriana than in Navel orange, consistent with the differing tolerance. Resistance (R) genes specifically activate a resistance reaction to a particular pathogen. NBS-LRR proteins are the most numerous R-gene class. NBS-LRR genes are finely controlled by regulatory mechanisms that allow their expression only when a biotic attack occurs, and limiting their metabolic cost when they are not required [40]. It is possible that the two NBS-LRR proteins upregulated in Volkameriana may contribute to enhanced tolerance of HLB disease. Heat shock proteins (HSP) are molecular chaperones with important functions in non-covalent protein folding or unfolding, assembly, and modifications. Genes encoding HSP70, HSP82 and other small heat shock proteins were expressed at lower levels in HLB disease in both fruit and leaf tissues [16, 41]. Down-regulation of HSP70, chaperon$60 \mathrm{kD}$ and chaperonin-60alpha was also seen in infected grapefruit [17]. In the present study, HSP81, HSP21 and HSP23 were induced in Volkameriana while several HSP proteins were inhibited in Navel orange. Taken together, we conclude that the observed upregulation of some HSPs in Volkameriana may contribute to increased tolerance to HLB disease. 


\section{Overall metabolism}

The repression of key proteins involved in photosynthetic light reactions was linked with the upregulation of starch-related pathways. Infected Volkameriana exhibited up-regulated nitrilases, oxidases, glutathione-Stransferases and other proteins involved in redox state. Infected Volkameriana also exhibited enhanced production of expansins and xyloglucan endotransglycosylases.

Proteins commonly altered by HLB in both genotypes strengthen the data at proteomic level. The WD40 repeatlike protein was upregulated along with some enzymes involved in protein targeting, degradation, and glycosylation such as cysteine peptidase 3 , proteinase A1, and cysteine protease. Additional file 5: Figure S3 presents a complete list of commonly regulated proteins and their abundance in the four examined Citrus sample categories.

\section{Conclusions}

Forty-six of the 71 proteins were encoded by genes that were found to be transcriptionally regulated by HLB by previous studies in field conditions. Any comparison between transcriptomic and proteomic studies should take into consideration that experiments were performed with different plant material grown under differing environmental, developmental and physiological conditions. We have applied an integrated approach using principal component analysis (PCA), gene set enrichment analysis and functional data mining to identify specific key proteomic changes in response to Huanglongbing disease in these two Citrus genotypes. The analysis of post-transcriptional mechanisms is an essential step to link molecular regulatory networks to observed phenotypic changes. Interestingly, the clearest differences between the two genotypes were observed for proteins involved in redox state and detoxification pathways such as glutathione-S-tranferases. HLB disease strongly affected genes and metabolites of the terpenoid, carotenoid, and jasmonic acid pathways [16, 42].

\section{Methods}

\section{Material and experimental design}

Citrus plant materials used in this study were propagated from disease free bud wood obtained from the California Citrus Clonal Protection Program (CCPP). Two-year old Volkameriana (V) (Citrus $\times$ volkameriana) and Navel orange $(\mathrm{N})$ (Citrus $\times$ sinensis) trees were grafted on Carrizo citrange rootstocks (Citrus sinensis [L.] Osb. $\times$ Poncirus trifoliata [L.] Raf.). Trees were grown in pots in the greenhouse under natural light at 17 to $25^{\circ} \mathrm{C}$ in the Contained Research Facility (CRF) at UC Davis. Around 10 trees per genotype were infected with CaLas through graft inoculations using a standard inverted " $\mathrm{T}$ " budding technique with infected budwood from Lisbon Lemon (Citrus limon Burm.f.), with uninocculated trees maintained as an uninfected control. Starting at 3 months after budding, each plant was tested monthly using quantitative RT-PCR for CaLas species as described [43]. Three to four biological replicates of healthy and infected symptomatic trees were chosen for proteomic analysis based on health, phenotype and symptom severity. A pool of five to seven fully expanded leaves at the same developmental stage from each tree was sampled at 8 months, constituting a biological replicate. From infected trees, leaves with characteristic yellowing and blotchy mottled appearance were sampled. Healthy leaves at the same developmental stage were harvested from the uninfected control trees. Petioles from four to six leaves sampled from different parts of each tree were tested by PCR for the presence of CaLas at the time of collection. Midribs and petioles were cut, frozen in liquid nitrogen and stored at $-80{ }^{\circ} \mathrm{C}$ for protein extraction and iTRAQ analysis. The other parts of the leaves were used to test for pathogen presence. Ct values of infected trees were $<30$ while control trees showed no amplified product.

\section{Protein extraction}

Proteins were extracted using a previously described phenol-based procedure [44]. Leaves were ground in a mortar and pestle in liquid nitrogen with $1 \%(\mathrm{w} / \mathrm{w})$ PVPP. One hundred mg plant material was resuspended in $600 \mu \mathrm{L}$ extraction buffer $(0.7 \mathrm{M}$ Sucrose, $0.1 \mathrm{M} \mathrm{KCl}$, $0.5 \mathrm{M}$ Tris- $\mathrm{HCl}$ pH7.5, 0.5 M EDTA, $1 \mathrm{mM}$ PMSF and $2 \% \beta$-mercaptoethanol). Samples were homogenized twice (one min each) with a MM300 TissueLyser (Qiagen). An equal volume of UltraPure Buffer-Saturated Phenol (Invitrogen) was added and the mixture was rehomogenized as described above. After centrifugation at 12,000 $\times \mathrm{g}$ for $15 \mathrm{~min}$ at $4{ }^{\circ} \mathrm{C}$, the upper phenol phase was eliminated and the pellet used for re-extraction in the same buffer. Protein was precipitated from the phenol phase using five volumes saturated ammonium acetate $(100 \mathrm{mM})$ in methanol overnight at $-20{ }^{\circ} \mathrm{C}$ followed by centrifugation at $12,000 \times \mathrm{g}$ for $15 \mathrm{~min}$ at $4{ }^{\circ} \mathrm{C}$. Pellets were washed four times with four $\mathrm{mL}$ saturated ammonium acetate $(100 \mathrm{mM})$ in methanol and dried $10 \mathrm{~min}$. Proteins were dissolved in urea buffer (7 M urea, $2 \mathrm{M}$ thiourea, $40 \mathrm{mM}$ Tris, $2 \%$ Chaps and $18 \mathrm{mM}$ DTT). The protein concentration was determined using Bradford's method with BSA as a standard.

\section{Protein sample preparation and digestion}

Samples were precipitated using the ProteoExtract Protein Precipitation Kit (CalBiochem). The resulting protein pellet was solubilized in $400 \mu \mathrm{L}$ of $50 \mathrm{mM}$ triethyl ammonium bicarbonate (TEAB) and a $100 \mu \mathrm{L}$ aliquot was taken for digestion. $500 \mathrm{mM}$ tris (2-carboxyethyl)phosphine (TCEP) (Pierce, Rockford, IL) was added to a final concentration of $10 \mathrm{mM}$ and samples were incubated for $10 \mathrm{~min}$ at $90{ }^{\circ} \mathrm{C}$ to reduce disulfide bonds. 
Next, $110 \mathrm{mM}$ iodoacetamide (IAA) was added to a final concentration of $15 \mathrm{mM}$ and incubated for $1 \mathrm{~h}$ at room temperature, followed by the addition of $20 \mu \mathrm{L}$ DTT to quench the IAA reaction. Trypsin (Promega) was next added in a 1:25 ratio (enzyme: protein) and incubated at $37{ }^{\circ} \mathrm{C}$ for overnight. The following day, samples were desalted using C18 Macro Spin columns (Nest Group) and dried down by vacuum centrifugation.

\section{Tandem mass tag labeling}

Desalted and lyophilized samples were resuspended in $50 \mathrm{mM}$ TEAB and $30 \mathrm{ug}$ of tryptic digested peptides were taken for TMT labeling. TMT labeling was performed on each aliquot with reporter ions $\mathrm{m} / \mathrm{z}=126.1$, $127.1,128.1$, and 129.1 in $41 \mu \mathrm{L}$ ethanol, and aliquots were incubated for $60 \mathrm{~min}$ at room temperature. $8 \mu \mathrm{L}$ hydroxylamine $5 \%(\mathrm{v}: \mathrm{v})$ was added to quench the reaction and samples were vacuum-centrifuged prior to desalting using C18 Macro Spin columns (Nest Group). Samples were vacuum-centrifuged once more prior to strong cation exchange fractionation.

\section{SCX fractionation of Pooled TMT-labeled samples}

Strong cation exchange (SCX) was carried out using the SCX SpinTips Sample Prep Kit (ProteaBio). Each aliquot was resuspended in $50 \mu \mathrm{L}$ of the designated buffer and $\sim 10 \mu \mathrm{g}$ of each sample was pooled prior to SCX fractionation. Samples were fractionated by stepwise addition of $20,40,60,80,100,150,250$, and $500 \mathrm{mM}$ ammonium formate in $10 \%$ acetonitrile. All eight fractions, including the initial binding flow through, were vacuum-centrifuged to remove any acetonitrile and desalted using C18 Macro Spin columns (Nest Group).

\section{LC-MS/MS analysis}

LC separation was done on a Waters Nano Acquity UHPLC (Waters Corporation) with a Proxeon nanospray source. Each SCX fraction (9 total) was reconstituted in $2 \%$ acetonitrile $/ 0.1 \%$ trifluoroacetic acid and loaded onto a $100 \mu \mathrm{m} \times 25 \mathrm{~mm}$ Magic C18 $100 \AA 5 \mathrm{U}$ reverse phase trap. Peptides were eluted using a gradient of 0.1 formic acid (A) and $100 \%$ acetonitrile (B) with a flow rate of $300 \mathrm{~nL} / \mathrm{min}$. A $60 \mathrm{~min}$ gradient was run with 5 to $35 \mathrm{~B}$ over $50 \mathrm{~min}, 35$ to $80 \mathrm{~B}$ over $3 \mathrm{~min}, 80 \mathrm{~B}$ for $1 \mathrm{~min}, 80$ to $5 \mathrm{~B}$ over $1 \mathrm{~min}$, and finally held at $5 \%$ B for $5 \mathrm{~min}$.

Mass spectra were collected on an Orbitrap Q Exactive Plus mass spectrometer (Thermo Fisher Scientific). A dynamic exclusion of $15 \mathrm{~s}$ was used. MS spectra were acquired with a resolution of 70,000 and a target of $1 \times 106$ ions or a maximum injection time of $30 \mathrm{~ms}$. MS/MS spectra were acquired with a resolution of 17,500 and a target of $5 \times 104$ ions or a maximum injection time of $50 \mathrm{~ms}$, and a fixed first mass of $110 \mathrm{~m} / \mathrm{z}$. Peptide fragmentation was performed using higher-energy collision dissociation with a normalized collision energy value of 30. Unassigned charge states as well as +1 and ions $>+5$ were excluded from MS/MS fragmentation.

\section{Data analysis}

Tandem mass spectra were extracted and charge states were deconvoluted and deisotoped. All MS/MS samples were analyzed using X! Tandem (The GPM, thegpm.org; version X! Tandem Sledgehammer (2013.09.01.1)). X! Tandem was set up to search the Citrus sinensis genome (http://www.ncbi.nlm.nih.gov/protein/?term=txid2 706 (March 2014) and the NCBInr citrus database $(155,237$ entries, March 2014) assuming the digestion enzyme trypsin. X! Tandem was searched with a fragment ion mass tolerance of 20 PPM and a parent ion tolerance of 20 PPM. TMT6plex of lysine and the n-terminus was specified in $\mathrm{X}$ ! Tandem as a fixed modification.

Scaffold Q+ (version Scaffold_4.4.0, Proteome Software Inc., Portland, OR) was used to quantitate Label Based Quantitation (iTRAQ, TMT, SILAC, etc.) peptide and protein identifications. Peptide identifications were accepted if they could be established at a $99.0 \%$ probability by the Scaffold Local FDR algorithm, which corresponded to a 0.20 spectra decoy FDR and a $5.0 \%$ protein decoy FDR with 1 identified peptides per protein. Protein probabilities were assigned by the Protein Prophet algorithm [45]. Proteins that contained similar peptides and could not be differentiated based on MS/MS analysis alone were grouped to satisfy the principles of parsimony. Proteins sharing significant peptide evidence were grouped into clusters. Proteins sharing significant peptide evidence were grouped into clusters according to the algorithm described in i-Tracker [46]. Individual quantitative samples were normalized within each acquisition run. Intensities for each peptide identification were normalized within the assigned protein. All normalization calculations were performed using medians to multiplicatively normalize data. Differentially expressed proteins were determined using Permutation Test analysis.

\section{Statistical and functional data mining}

Scaffold 4 was used to perform the first functional, annotation and quantitative analysis of the proteomic data. Arabidopsis orthologs, annotations, unique peptides and spectrum counts, and normalized quantitative values were determined for each sequenced and identified peptide. Data were blasted against the Citrus $x$ sinensis (L.) and Candidatus liberibacter asiaticus (strain psy62) genomes. Arabidopsis orthologs were determined for each sequenced peptide by blastx $(\mathrm{e}$-value $<10-4)$ to the TAIR database of predicted proteins in Arabidopsis (TAIR10_- pep_20101028; [47]). Blastx output was processed using custom scripts to calculate the best correspondence between individual citrus peptide sequences 
and Arabidopsis proteins, based on alignments over the entire length of each sequence. Lists of significantly differentially expressed proteins $(p<0.05$, absolute value of $\log 2$ fold change $>0.5$ or $<-0.5$ ) were determined in pairwise comparison (infected/healthy) for each genotypes. This statistical analysis was performed using $\mathrm{MeV}$ software. Functions of differentially regulated proteins (as Arabidopsis orthologs) were visualized using MapMan [48]. Gene set enrichment analysis was performed using DAVID Bioinformatics resource 6.7 based on KEGG maps. The corresponding Arabidopsis orthologs of each protein upregulated or downregulated during infection for each genotype was loaded as a gene list in DAVID $(p<0.05)$. Arabidopsis orthologs were determined for each citrus protein and the gene set enrichment analysis was obtained comparing the list of differentially regulated proteins with all those identified by the proteomic analysis.

The PageMan visualization tool was used for GSEA with the Wilcoxon test (no correction and 1.0 as ORA cutoff). Principal component analysis (PCA) was performed using SAS II (2008) SAS/STAT software (SAS Institute). Principal component analysis was applied to the ratio matrix of gene expression data to examine the contribution of each target gene to the separation of sample classes. A biplot was constructed based on the first two principal components.

\section{Additional files}

Additional file 1: Table S1. Differentially regulated host proteins in control and infected leaf tissues of Navel orange ( $p$-value $<0.05$ ). Arabidopsis orthologs and log fold ratio are indicated. (XLS $67 \mathrm{~kb}$ )

Additional file 2: Table S2. Differentially regulated host proteins in control and infected leaf tissues of Volkameriana ( $p$-value $<0.05$ ). Arabidopsis orthologs and log fold ratio are indicated. (XLS $55 \mathrm{~kb}$ )

Additional file 3: Figure S1. Metabolism oveview of proteomic changes in response to HLB disease, comparing effects in the two Citrus genotypes. (PDF $115 \mathrm{~kb}$ )

Additional file 4: Figure S2. HLB-differentially regulated proteins involved in secondary metabolism in the two Citrus genotypes. Each colored square represents the expression change (see color key) in a protein associated with the biosynthetic pathway. (TIF $268 \mathrm{~kb}$ )

Additional file 5: Figure S3. A list of commonly regulated proteins by $H L B$, comparing effects in the two Citrus genotypes. $V C=$ Volkameriana control (healthy), $\mathrm{Vl}=$ Volkameriana Infected, NC = Navel orange control (healthy), $\mathrm{Nl}=$ Navel orange infected. Annotation and Citrus ID are indicated. (PDF $121 \mathrm{~kb}$ )

\footnotetext{
Abbreviations

2-DE, Two-dimensional electrophoresis; ABC transporters, ATP-binding cassette transporters; ADPase, ADP glucose-pyrophospholyase; BAK1, BCL2-Antagonist/ Killer 1; CaLas, Candidatus Liberibacter asiaticus; CCPP, Citrus Clonal Protection Program; CRF, Contained Research Facility; CYP711A1, cytochrome P450, family 711, subfamily A, polypeptide 1; DMAPP, Dimethylallyl pyrophosphate; DUF26, Domain of Unknown Function 26; ET, Ethylene; GASA1, GAST1 protein homolog 1; GPT2, Glucose-phosphate-transporter 2; GSTS, Glutathione S-transferases; HLB, Huanglongbing; HSP, Heat shock proteins; IAA, Indole 3 acetic acid; ICP-MS, Inductively coupled plasma mass spectrometry; IPP, Isopentenyl pyrophosphate; ITRAQ, Isobaric tags for relative and absolute quantitation; JA, Jasmonic acid; LRR, Leucine rich repeats; MVA, mevalonate pathway; N, Navel orange; NBS-LRR,
}

Nucleotide-binding site-leucine-rich repeat; PAL, Phenylammonialyase; PAMP, Pathogen-associated molecular patterns; PCA, Principal component analysis; PEP, phospho-enol-pyruvate; PPM, Parts-per-million; RHM1, UDP-L-rhamnose synthase; RLKs, receptor-like kinases; SA, Salicilic acid; SAUR, small auxin-up RNA; SCX, Strong cation exchange; TCA cycle, Tricarboxylic acid cycle; TCEP, Tris (2-carboxyethyl)-phosphine; TCH4, xyloglucanxyloglucosyltransferase; TEAB, Triethyl ammonium bicarbonate; TMT labeling, Tandem mass tags; UXS2, UDP-glucuronic acid decarboxylase 2; UXS6, UDP-glucuronic acid decarboxylase 6; V, Volkameriana

\section{Acknowledgements}

The authors wish to thank Kristine Godfrey at the Contained Research Facility for her help with the infected plant materials, Brett S Phinney and Darren Webber at Proteomics Core facility UCD Genome Center for performing the proteomic analysis, Ana Maria Ibanez, UCD Plant Sciences Department for her advice during the protein extraction phase and Mary Lou Mendum for her editorial review of the manuscript.

\section{Funding}

The work was supported by grants obtained from the Citrus Research and Development Foundation.

\section{Availability of data and materials}

Data were provided in Additional files 1 and 2: Tables S1 and S2.

\section{Authors' contributions}

AMD and FM conceived and designed the study. DD developed the plant materials. FM and DD carried out the proteomic experiments. FM, RLR and VF designed the figures and contributed to the statistical analysis. AMD coordinated all the work. All authors read and approved the final manuscript.

\section{Competing interests}

The author(s) declare that they have no competing interests.

\section{Consent for publication}

All authors consent to publish all contents included in the present work. Our manuscript does not contain any individual's personal data, and therefore it belongs to the category "Not applicable".

\section{Ethics approval and consent to participate}

The work does not involve any research on humans or animals. Experimental research reported here complied with institutional, national, or international guidelines. The study has been conducted with samples present in the Contained Research Facility at UC Davis, a quarantine facility following all related Federal and local guidelines, and with approvals and permits obtained from federal agencies and with the project approvial from the local institutional biosafety committee.

We have obtained the appropriate permissions and/or licenses to conduct this study and it does not involve species included in the Convention on the Trade in Endangered Species of Wild Fauna and Flora.

\section{Author details}

'Department of Agricultural and Forest Sciences, University of Palermo, viale delle scienze ed. 4, 90128 Palermo, Italy. ${ }^{2}$ Plant Sciences Department, University of California, One Shields Avenue, 95616 Davis, CA, USA.

Received: 25 February 2016 Accepted: 20 July 2016 Published online: 28 July 2016

\section{References}

1. Bové JM. Huanglongbing: a destructive, newly-emerging, century-old disease of Citrus. J Plant Pathol. 2006;88:7-37.

2. Folimonova SY, Achor D. Early events of Citrus greening (Huanglongbing) disease development at the ultrastructural level. Bacteriology. 2010;100:949-58.

3. Jagoueix S, Bové JM, Garnier M. The phloem-limited bacterium of greening disease of Citrus is a member of the alpha subdivision of the Proteobacteria. Int J of Syst Bacteriol. 1994:44:397-86.

4. Sechler A, Schuenzel EL, Cooke P, Donnua S, Thaveechai N, Postnikova E, Stone AL, Schneider WL, Damsteegt VD, Schaad NW. Cultivation of "Candidatus Liberibacter asiaticus", "Ca. L. africanus", and "Ca. L. americanus" associated with huanglongbing. Phytopathology. 2009;99:480-86. 
5. Folimonova SY, Robertson CJ, Garnsey SM, Gowda S, Dawson WO. Examination of the responses of different genotypes of Citrus to Huanglongbing (Citrus greening) under different conditions. Phytopathology. 2009;99:1346-54.

6. Fraser $L R$, Singh $D$. Reaction of Indian citrus varieties to greening virus. In: Chapman HD, editor. Proc. 1st Int. Citrus Symp. Vol. I, Univ. Calif., Riverside. 1969. p. 365-6.

7. Manjunath KL, Halbert SE, Ramadugu C, Webb S, Lee RF. Detection of 'Candidatus Liberibacter asiaticus' in Diaphorina citriand its importance in the management of Citrus huanglongbing in Florida. Phytopathology. 2008;98:387-96.

8. Ramadugu C, Keremane ML, Halbert SE, Duan Y, Roose M, Stover E, Lee RF. Long term field evaluation reveals HLB resistance in Citrus relatives. Plant Dis. http://dx.doi.org/10.1094/PDIS-03-16-0271-RE.

9. McCollum G, Hilf M. Susceptibility of Sixteen Citrus Genotypes to 'Candidatus Liberibacter asiaticus'. Plant Dis. 2016:100:1080-6.

10. Albrecht $\mathrm{U}$, Fiehn O, Bowman KD. Metabolic variations in different citrus rootstock cultivars associated with different responses to Huanglongbing. Plant Physiol. Biochem. 2016;107:33-44.

11. Tyler HL, Roesch LFW, Gowda S, Dawson WO, Triplett EW. Confirmation of the sequence of 'Candidatus liberibacter asiaticus' and assessment of microbial diversity in huanglongbing-infected Citrus phloem using a metagenomic approach. Mol Plant Microbe. 2009;22:1624-34.

12. Duan Y, Zhou L, Hall DG, Li W, Doddapaneni H, Lin H, Liu L, Vahling CM, Gabriel DW, Williams KP, Dickerman A, Sun Y, Gottwald T. Complete genome sequence of Citrus huanglongbing bacterium, 'Candidatus Liberibacter asiaticus' obtained through metagenomics. MPMI. 2009;22:1011-20.

13. Albrecht $\mathrm{U}$, Bowman $\mathrm{KD}$. Gene expression in Citrus sinensis (L.) Osbeck following infection with the bacterial pathogen Candidatus Liberibacter asiaticus causing Huanglongbing in Florida. Plant Sci. 2008;175:291-306.

14. Kim J-S, Sagaram US, Burns JK, Li J-L, Wang N. Response of sweet orange (Citrus sinensis) to 'Candidatus Liberibacter asiaticus' infection: microscopy and microarray analyses. Phytopathology. 2009:99:50-7.

15. Martinelli F, Uratsu SL, Albrecht U, Reagan RL, Phu ML, Britton M, Buffalo V, Fass J, Leicht E, Zhao W, Lin D, D'Souza R, Davis CE, Bowman KD, Dandekar AM. Transcriptome profiling of Citrus fruit response to Huanglongbing disease. PLoS One. 2012:7:e38039.

16. Martinelli F, Reagan RL, Uratsu SL, Phu ML, Albrecht U, Zhao W, Davis CE, Bowman KD, Dandekar AM. Gene regulatory networks elucidating huanglongbing disease mechanisms. Plos One. 2013;8:e74256.

17. Nwugo CC, Lin H, Duan Y, Civerolo EL. The effect of 'Candidatus Liberibacter asiaticus'infection on the proteomic profiles and nutritional status of pre-symptomatic and symptomatic grapefruit (Citrus paradisi) plants. BMC Plant Biol. 2013;13:59.

18. Fan J, Chen C, Yu Q, Brlansky RH, Li Z-G, Gmitter FG. Comparative iTRAQ proteome and transcriptome analyses of sweet orange infected by "Candidatus Liberibacter asiaticus". Physiol Plant. 2011;143:235-45.

19. Cevallos-Cevallos JM, García-Torres R, Etxeberria E, Reyes-De-Corcuera Jl. GC-MS analysis of headspace and liquid extracts for metabolomic differentiation of citrus huanglongbing and zinc deficiency in leaves of 'Valencia' sweet orange from commercial groves. Phytochem Anal. 2011;22:236-46.

20. Cevallos-Cevallos JM, Futch DB, Shilts T, Folimonova SY, Reyes-De-Corcuera J. GC-MS metabolomic differentiation of selected Citrus varieties with different sensitivity to citrus huanglongbing. Plant Physiol Biochem. 2012;53:69-76.

21. Killiny N, Hijaz F. Amino acids implicated in plant defense are higher in Candidatus Liberibacter asiaticus-tolerant citrus varieties. Plant Signal Behav. 2016:4:e1171449.

22. Nakamura T, Yamamori M, Hirano H, Hidaka S, Nagamine T. Production of waxy (amylose-free) wheats. MGG Mol Gen Gen. 1995;248:253-59.

23. Tauzin AS, Giardina T. Sucrose and invertases, a part of the plant defense response to the biotic stresses. Front Plant Sci. 2014;5:293.

24. Herbers K, Takahata Y, Melzer M, Mock HP, Hajirezaei M, Sonnewald U. Regulation of carbohydrate partitioning during the interaction of potato virus Y with tobacco. Mol Plant Pathol. 2000;1:51-9.

25. Cheynier V, Comte G, Davies KM, Lattanzio V, Martens S. Plant phenolics: recent advances on their biosynthesis, genetics, and ecophysiology. Plant Physiol Biochem. 2013;72:1-20.

26. Tonelli ML, Ibanez F, Taurian T, Argüello J, Fabra A. Analysis of a phenylalanine ammonia-lyase gene sequence from Arachis hypogaea $\mathrm{L}$. and its transcript abundance in induced systemic resistance against Sclerotium rolfsii. J Plant Pathol. 2013;95:191-95.
27. Nakashita H, Yasuda M, Nitta T, Asami T, Fujioka S, Arai Y, Sekimata K, Takatsuto S, Yamaguchi I, Yoshida S. Brassinosteroid functions in a broad range of disease resistance in tobacco and rice. Plant J. 2003;33:887-98.

28. Chinchilla D, Zipfel C, Robatzek S, Kemmerling B, Nürnberger T, Jones JDG, Felix G, Bolle T. A flagellin-induced complex of the receptor FLS2 and BAK1 initiates plant defence. Nature. 2007:448:497-500.

29. Pieterse CMJ, Leon-Reyes A, Van der Ent S, Van Wees SCM. Networking by small-molecules hormones in plant immunity. Nat Chem Biol. 2009;5:308-16.

30. Feuillet C, Schachermayr G, Keller B. Molecular cloning of a new receptor-like kinase gene encoded at the Lr10 disease resistance locus of wheat. Plant J. 1997;11:45-52.

31. Jahn T, Fuglsang AT, Olsson A, Brüntrup IM, Collinge DB, Volkmann D, Sommarin M, Palmgren MG, Larsson C. The 14-3-3 protein interacts directly with the C-terminal region of the plant plasma membrane HC-ATPase. Plant Cell. 1997:9:1805-14.

32. Sheehan D, Meade G, Foley VM, Dowd CA. Structure, function and evolution of glutathione transferases: implications for classification of non-mammalian members of an ancient enzyme superfamily. Biochem J. 2001;360:1-16.

33. Edwards R, Dixon DP, Walbot V. Plant glutathione S-transferases: enzymes with multiple functions in sickness and in health. Trends Plant Sci. 2000;5:193-98.

34. Marrs KA. The functions and regulation of glutathione S-transferases in plants. Annu Rev Plant Physiol Plant Mol Biol. 1996;47:127-58.

35. Shigeoka S, Ishikawa T, Tamoi M, Miyagawa Y, Takeda T, Yabuta Y, Yoshimura K. Regulation and function of ascorbate peroxidase isoenzymes. J Exp Bot. 2002;53:1305-19.

36. Teixeira FK, Menezes-Benavente L, Galvão VC, Margis R, Margis-Pinheiro M. Rice ascorbate peroxidase gene family encodes functionally diverse isoforms localized in different subcellular compartments. Planta. 2006; 224:300-14.

37. Albrecht U, Bowman KD. Transcriptional response of susceptible and tolerant citrus to infection with 'Candidatus Liberibacter asiaticus'. Plant Sci. 2012:185-186:118-30.

38. Valtaud C, Larignon P, Roblin G, Fleurat-Lessard P. Developmental and ultrastructural features of Phaeomoniella chlamydospora and Phaeoacremonium aleophilum in relation to xylem degradation in esca disease of the grapevine. J Plant Pathol. 2009;91:37-51.

39. Lionetti V. PECTOPLATE: The simultaneous phenotyping of pectin methylesterases, pectinases, and oligogalacturonides in plants during biotic stresses. Front Plant Sci. 2015;6:1-8.

40. Marone D, Russo MA, Laidò G, De Leonardis AM, Mastrangelo AM. Plant Nucleotide Binding Site-Leucine-Rich Repeat (NBS-LRR) genes: active guardians in host defense responses. Int J Mol Sci. 2013;14:7302-26.

41. Martinelli F, Ibanez AM, Reagan RL, Phu M, Dandekar AM. Stress responses in citrus peel: Comparative analysis of host responses to Huanglongbing disease and puffing disorder. Sci Hortic. 2015b;192:409-20.

42. Dandekar AM, Martinelli F, Davis CE, Bhushan A, Zhao W, Fiehn O, Skogerson K, Wohlgemuth G, D'Souza R, Roy S, Reagan RL, Lin D, Cary RB, Pardington P, Gupta G. Analysis of early host responses for asymptomatic disease detection and management of specialty crops. Crit Rev Immunol. 2010;30:277-89.

43. Li W, Hartung JS, Levy L. Quantitative real-time PCR for detection and identification of Candidatus Liberibacter species associated with citrus huanglongbing. J Microbiol Methods. 2006;66:104-15.

44. Schuster AM, Davies E. Ribonucleic acid and protein metabolism in Pea epicotyls. Plant Physiol. 1983;73:817-21.

45. Shadforth IP, Dunkley TP, Lilley KS, Bessant C. i-Tracker: for quantitative proteomics using iTRAQTM. BMC Genomics. 2005;6:145.

46. Nesvizhskii A, Keller A, Kolker E, Aebersold R, Nesvizhskii Al, Keller A, Kolker E, Aebersold R. A statistical model for identifying proteins by tandem mass spectrometry. Anal Chem. 2003;75:4646-58.

47. Lamesch P, Berardini TZ, Li D, Swarbreck D, Wilks C, Sasidharan R, Muller R, Dreher K, Alexander DL, Garcia-Hernandez M, Karthikeyan AS, Lee CH, Nelson WD, Ploetz L, Singh S, Wensel A, Huala E. The Arabidopsis Information Resource (TAIR): improved gene annotation and new tools. Nucleic Acids Res. 2012:40(Database issue):D1202-10.

48. Thimm O, Bläsing O, Gibon Y, Nagel A, Meyer S, Krüger P, Selbig J, Müller $L A$, Rhee SY, Stitt M. MAPMAN: a user-driven tool to display genomics data sets onto diagrams of metabolic pathways and other biological processes. Plant J. 2004;37:914-39. 